\title{
Water Availability: A Hydrological View
}

\subsection{Global Water Resources and Water Cycle}

The whole amount of water on earth was generated during the earliest earth ages by volcanoes that emitted water vapor. Currently the amount of water which is allocated to the oceans, glaciers, polar ice, groundwater, lakes, and rivers stays nearly at a constant level.

The volume of the total water reserves is about 1,386 million $\mathrm{km}^{3}$ (Table 2.1). The major part of these water reserves (about 96.5\%) is located in the oceans as salt water. The total volume of freshwater stocks add up to 35 million $\mathrm{km}^{3}$, or just $2.5 \%$ of the total stock in the hydrosphere. A large fraction of freshwater (about 24 million $\mathrm{km}^{3}$ or $68.7 \%$ of freshwater stock) is stored in the Arctic and Antarctic regions in the form of ice and permafrost. About one-third of freshwater reserves are located in the aquifers as groundwater. Freshwater lakes and rivers, which are the most important sources for human water needs, contain on average about $90,000 \mathrm{~km}^{3}$, or $0.26 \%$ of total freshwater reserves (Shiklomanov 1990).

Atmospheric water in the form of vapor and clouds has a volume of about $12,900 \mathrm{~km}^{3}$, or $0.04 \%$ of total freshwater reserves. This atmospheric water is of high importance for the water cycle despite its small volume. If the atmospheric water precipitated completely, the water layer on the surface would have a height of just $25 \mathrm{~mm}$. However, the annual precipitation amount is about $1,000 \mathrm{~mm}$ which means that the whole water stock in the atmosphere regenerates every 10 days. All other types of water also renew, but the rates of renewal differ. For instance water in the rivers regenerates every 16 days on average, but the renewal period of glaciers, groundwater, ocean water, and the largest lakes run to hundreds or thousands of years (Shiklomanov 1990).

The "regeneration" of water in rivers, lakes, atmosphere, etc., is based on the conversion of water into different types and aggregate states. Water converts from one form to another and moves to various places, for instance, from the ocean to land and 
Table 2.1 Water availability on earth. Source Shiklomanov (1990)

\begin{tabular}{c|l|l|l}
\hline Source & Volume $\left[10^{3} \mathrm{~km}^{3}\right]$ & $\begin{array}{l}\text { Percent of total water } \\
{[\%]}\end{array}$ & $\begin{array}{l}\text { Percent of fresh water } \\
{[\%]}\end{array}$ \\
\hline Total water reserves & $1,385,984$ & 100 & - \\
\hline Total seawater & $1,338,000$ & 96.5 & - \\
\hline Total groundwater & 23,400 & 1.7 & - \\
\hline Soil moisture & 16,5 & 0.001 & 0.05 \\
\hline Freshwater & 10,530 & 0.76 & 30.1 \\
\hline Glaciers and permanent & 24,064 & 1.74 & 68.7 \\
\hline snow cover & & & 61.7 \\
\hline Antarctic & 21,600 & 1.56 & 6.68 \\
\hline Greenland & 2,340 & 0.17 & 0.24 \\
\hline Arctic islands & 83,5 & 0.006 & 0.12 \\
\hline Mountainous regions & 40,6 & 0.003 & 0.86 \\
\hline Ground ice/ permafrost & 300 & 0.022 & - \\
\hline Water reserves in lakes & 176,4 & 0.013 & 0.26 \\
\hline Fresh & 91 & 0.007 & - \\
\hline Saline & 85,4 & 0.006 & 0.03 \\
\hline Swamp water & 11,47 & 0.0008 & 0.006 \\
\hline River flows & 2,12 & 0.0002 & 0.003 \\
\hline Biological water & 1,12 & 0.0001 & 0.04 \\
\hline Atmospheric water & 12,9 & 0.001 & 100 \\
\hline Total freshwater reserves & 35,029 & 2.53 & \\
\hline & & & \\
\hline
\end{tabular}

back under the influence of solar energy and gravity. An overall diagram of the global water cycle is presented in Fig. 2.1. A large amount of water, about $505,000 \mathrm{~km}^{3}$, evaporates annually from the oceans' surface. About $90 \%$ of this evaporated amount, which is equal to about $458,000 \mathrm{~km}^{3}$, returns directly back to the oceans in the form of precipitation while $10 \%$ of this evaporated amount, which is equal to about $50,500 \mathrm{~km}^{3}$, precipitates on the land side. Together with evaporation and transpiration from land (about $68,500 \mathrm{~km}^{3}$ ), the total precipitation falling on dry land and supplying all types of land water is $119,000 \mathrm{~km}^{3}$. Based on this water volume, about $47,000 \mathrm{~km}^{3}$ per year is returned back to the oceans from land in the form of rivers, ground, and glacial run-off. On the whole about $577,000 \mathrm{~km}^{3}$ of water precipitates and evaporates on the earth. Thus, the world water balance can be considered as a closed system, such that

$$
P=E T=577000 \mathrm{~km}^{3}
$$

with: $P \ldots$ precipitation, $E T$...evapotranspiration

Figure 2.2 illustrates the levels of the main components of the global water circulation (Shiklomanov 1990). 


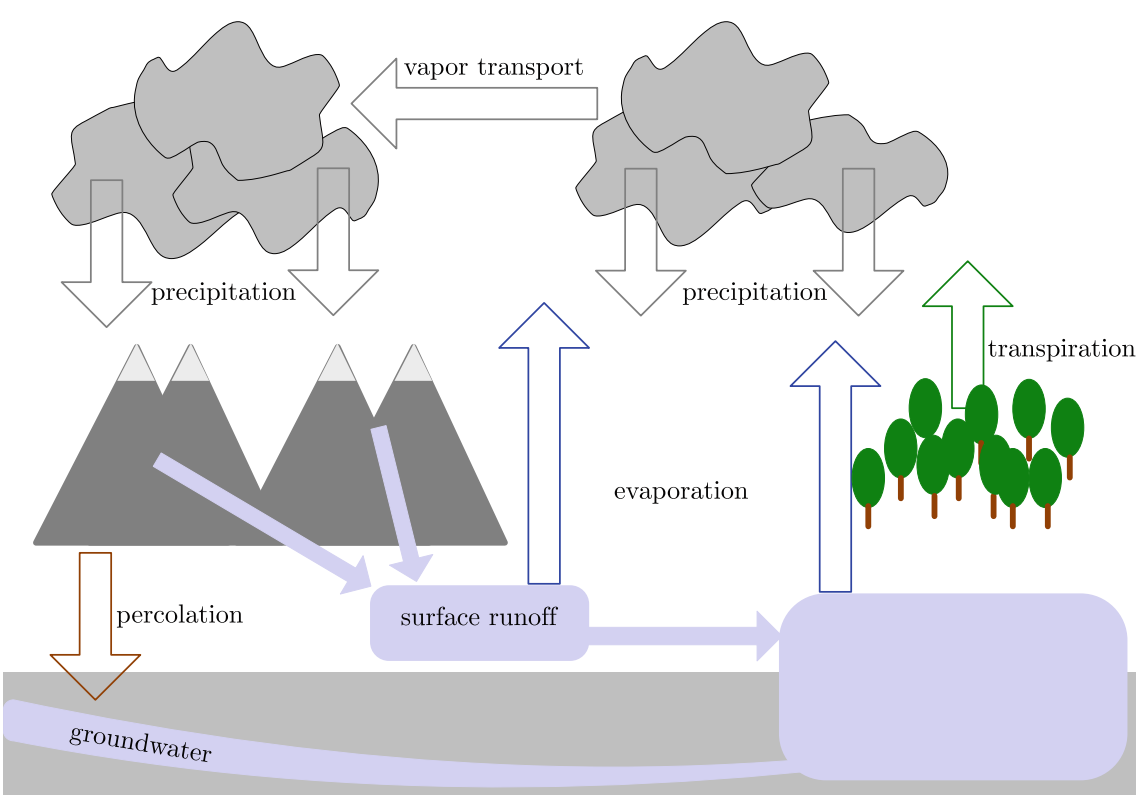

Fig. 2.1 Qualitative illustration of water cycle. Source adapted from Houghton (2004)

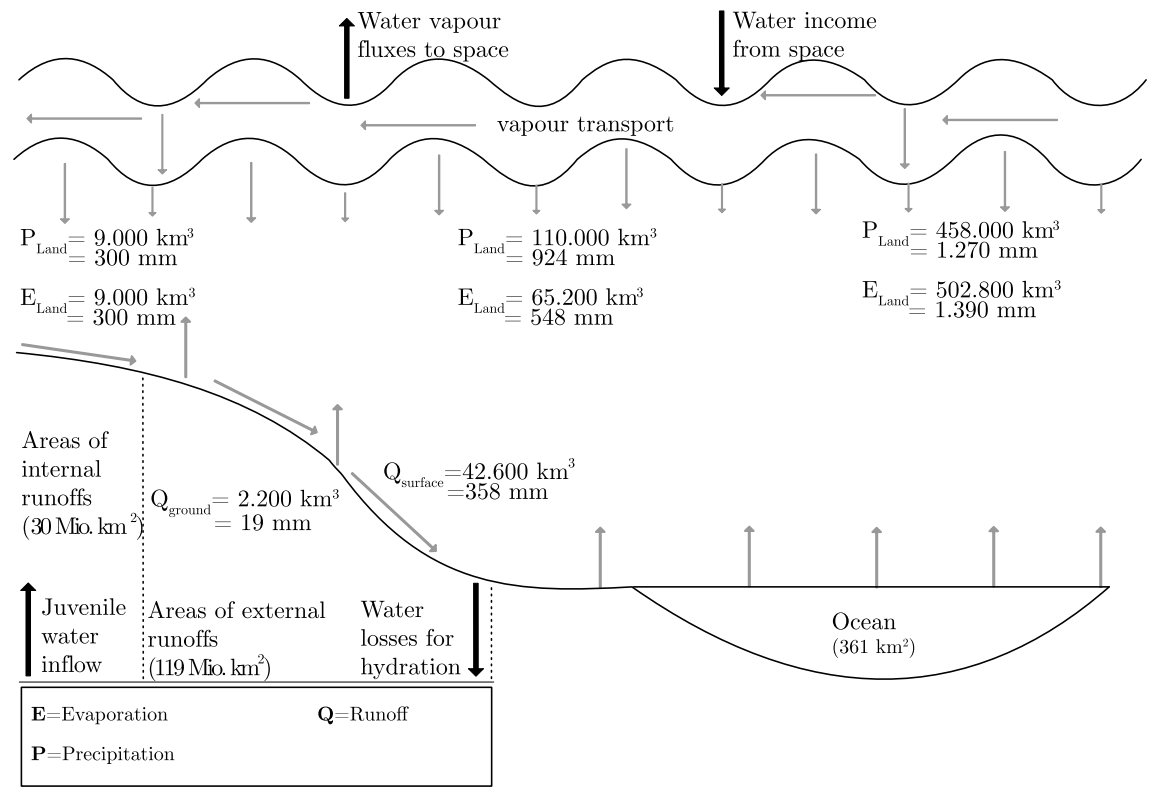

Fig.2.2 Levels of the main components of the global water cycle. Source adapted from Shiklomanov (1990) 


\subsection{The Regional Water Cycle}

Air humidity, soil surface, soil moisture storage, surface water (rivers and lakes), and groundwater are the types of water stocks that exist in each catchment. The relations and interconnections between these stocks are presented in Fig. 2.3. An external account is also introduced to illustrate the interconnection with the neighboring catchments. If water moves from a neighboring catchment to the considered catchment, the amount of water will increase in the addressed catchment. For instance, water vapor import fluxes induce an increase in air humidity; external recharges raise the amount of water in the aquifers and surface water stocks, etc. In contrast, the amount of water will decline in the respective stocks if water moves in the form of water vapor or surface and subsurface flows to neighboring catchments.

Water exchanges between the different water stocks also occur within the considered catchment. These exchanges and interconnections between the stocks are important to renew the stocks and to maintain the regional water cycle.

Precipitation, including all water in a hard or liquid state that reaches the soil surface from the atmospheric water stock (air humidity), is a very important input for plant, animal, and human life on earth. It will usually occur if the vapor pressure exceeds the saturated vapor pressure in the atmosphere. Falling precipitation, such as rainfall or snow, is usually known and the quantitatively most important kind of precipitation. Precipitation is a discontinuous and intermittent phenomenon with a high spatial and temporal variability. It is possible to distinguish between various forms of falling precipitation:

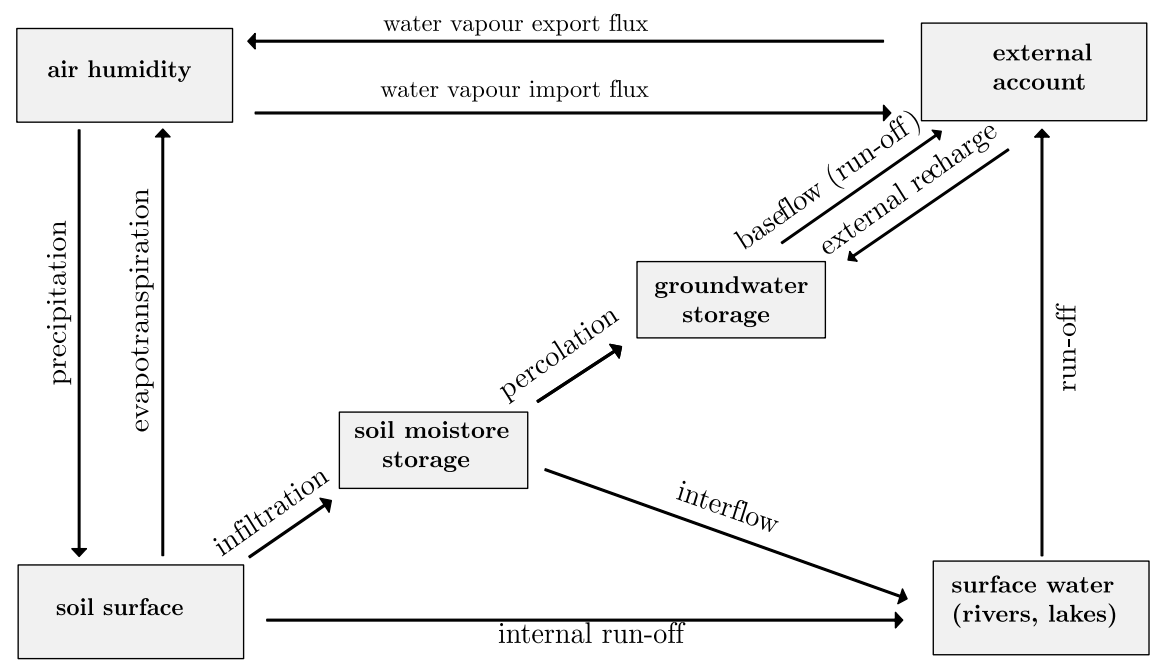

Fig. 2.3 Regional water cycle 
- Convective precipitation is characterized by a high intensity, short duration, smallscale appearance, and therefore, high temporal and spatial variability. In Europe, it usually occurs in the summer months in the form of heavy rainfall and small-scale thunderstorms.

- Advective precipitation (steady rain) is more continuous than convective rainfall. It is characterized by a large-scale extent, long duration, low or medium rain intensity, and relatively low spatial and temporal variability.

- The third type of falling precipitation is the orographic one that occurs on the windward side of a mountain and is caused by rising air masses that cool down and condensate. Orographic precipitations are characterized by a long duration and a large-scale extent on the windward side.

Besides the falling precipitation, which is well known, disposing ones, such as dew, rime, and frost also exist.

Another important phenomenon that influences the available liquid water resources in a considered basin is the evapotranspiration. The atmosphere and the hydrosphere of a basin are closely linked to the existence of precipitation and evapotranspiration, because a share of liquid water, which is fed to the stocks by external inflows or precipitation, is removed by evapotranspiration, a vaporization process of water. The potential evapotranspiration, which is a hypothetical value that expresses the maximum possible amount of water that could be vaporized, depends on various meteorological conditions, such as solar energy supply, temperature, humidity, and wind. While potential evapotranspiration assumes optimal water supply, the level of real evaporation is equal to the actual vaporized water under actual water supply conditions. Therefore, the level of calculated potential evapotranspiration exceeds the amount of water vaporized by real evapotranspiration. This total real evapotranspiration includes the sum of the evaporation, transpiration, and interception. Evaporation is a pure physical process and occurs only on the surface of water and bare soil. Therefore, this kind of water vaporization influences the stored water volumes on the surface soil and surface water resources (lakes and rivers) as well as the moisture in the soil.

Evaporation accounts for only 10-15\% of evapotranspiration in Central Europe while this proportion is much higher in arid regions because of less vegetation and higher solar energy supply. In Central Europe, the majority of the real evapotranspiration (about 70-75\%) is related to transpiration, which is a biological process in which water vapor is released by parts of the plants. 90-95\% of transpired water is released by the plants' stomata while the residual proportion is released by the cuticle. The transpiration can be regulated by opening and closing the stomata to prevent dehydration of the plant. Therefore, real evapotranspiration can deviate from potential evapotranspiration especially during hot spells. The third kind of evapotranspiration is the interception, which accounts for about $15 \%$ of total real evapotranspiration in Central Europe: It occurs on the surface of the plant; however, it is a pure physical process which cannot be influenced by the plant. Therefore, interception is often assigned to the evaporation. 
Because of interception and evaporation, the quantity of surface and subsurface runoff is lower than total precipitation. The share of liquid precipitation that is not evaporated directly usually becomes surface or subsurface runoff. In contrast to precipitation and runoff which is characterized as blue water, water which is vaporized by transpiration is classified as green water. The definition of blue and green water is explained in Box 2.1. Groundwater recharge occurs if seeped water reaches the groundwater stock. The groundwater is that kind of water that completely fills all cavities in the underground and whose movement is only based on gravity. The level of groundwater recharge in a basin mainly depends on the level of precipitation, solar radiation, ground utilization, ground properties, and the distance between aquifers and surface. Infiltrated water can also drain as an interflow next to the soil surface. If seeped water does not reach an aquifer the subsurface runoff is referred to as interflow.

\section{Box 2.1 Blue and green water}

Water that is directly used for biomass production and "lost" in evaporation is termed "green water", while "blue water" is the flowing water in surface water bodies (e.g., rivers, lakes) and subsurface water bodies (aquifers). Terrestrial ecosystems (e.g., crops) are often "green water" dependent while aquatic systems are often "blue water" dependent. The management of "green water" flows holds potentials for saving water.

Source: GWP (2000)

Human activities significantly impact the water cycle. Both the quality and quantity of water stocks are influenced by discharged wastewater, climate change and water abstraction. Figure 2.4 integrates several human activities in the natural water cycle. Abstractions from the groundwater and surface water body are necessary to cover the agricultural, domestic, and industrial water demand. Wastewater that occurs after the usage of freshwater will eventually be purified in the sewage plant. The purified or non-purified wastewater will be discharged in surface water or groundwater bodies by percolation subsequently. This discharge changes the quality of water in the water stocks.

\subsection{A Simplified Hydro-Economic Model}

Water management is only possible on the basis of an exact consideration of the complex relationships of the water cycle. This section introduces the basic elements of the water cycle and relates them to the water use of the economy. It is important to understand the circulatory character of water. In the following, more complex nonlinear relationships that have been developed in hydrology, play no role in the analysis 


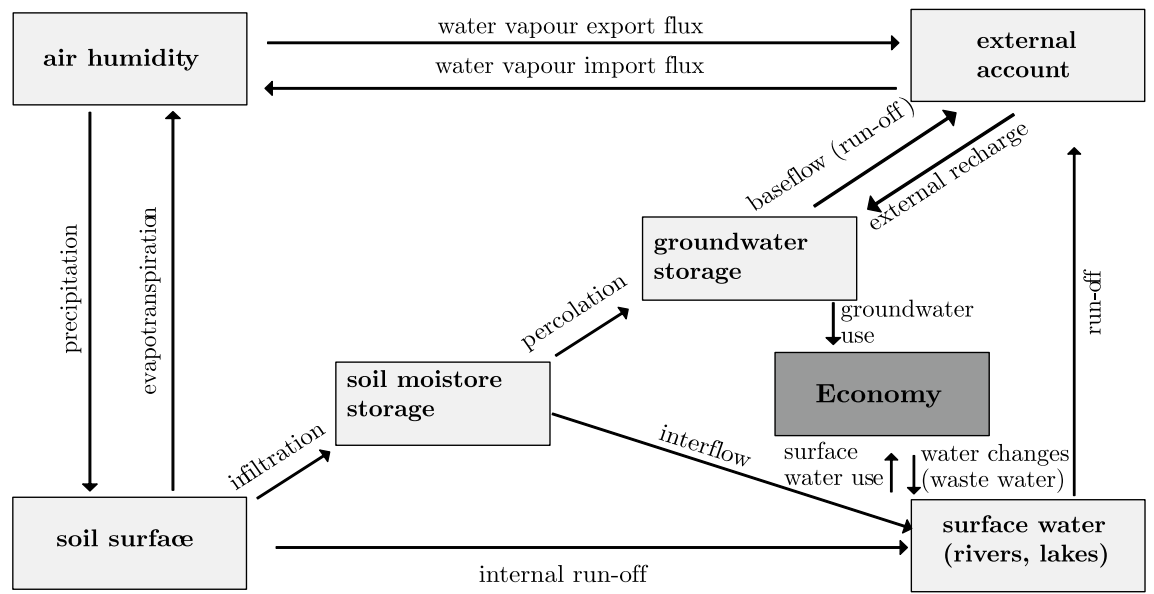

Fig. 2.4 Regional water cycle with human economy. Source own illustration

presented here at first. On the basis of a simple hydrological model, conclusions can be drawn which are presented in the following chapters.

A water cycle in its simplest form can be characterized by the dynamic mass balance equation, which describes the development of a water stock, including groundwater, water volume of surface water, etc., over time

$$
\frac{d S(t)}{d t}=R(t)+P(t)-E T(t)-(1-h) \cdot x(t)-r(t)
$$

In the balance equation, depicted in Eq. (2.1), the volume of the water stock at time $t$ is denoted by $S(t)$. The water can be the groundwater under a catchment area, a lake or the water volume of a river. ${ }^{1} R(t)$ and $P(t)$ stand for recharge and precipitation, respectively. Both variables are taken as exogenous, i.e., they are not determined by the water management of the economy of that catchment area. Recharge may happen by a river entering the area or by subterranean groundwater flows from outside. The same applies to precipitation. Rain comes with the wind into an area and is as such exogenously given. Of course, a certain proportion of the rain can also have arisen through the local water cycle. $x$ denotes the amount of water used in the local economy. The parameter $h \in[0,1]$ gives the portion of $x$ that is returned into the local watershed. ${ }^{2}$ For simplicity, we take $R, P$, and $x$ as time-independent. $r(t)$ describes the runoff at time $t$. Runoffs are all streams, be it on surface or underground, that leave the area. They depend, of course, on the water management of the economy and on the hydrology of the catchment area. $E T(t)$ depicts evapotranspiration. It

\footnotetext{
${ }^{1}$ The humidity of the soil also plays a role, but it is not considered in the following simplified model. ${ }^{2}$ Notice that we do not include water quality aspects into this basic model. Section 3.10 deals with water quality management.
} 
consists of that portion of water that leaves the area as vapor. Forests, plants, and crops transpire and water evaporates on the surface of the landscape. This green water rises up and is carried with the wind in various directions. A part of it returns as rain.

To keep the model as simple as possible, we assume linearity of the various interrelations between the variables of the hydrological cycle. In the following, we assume that evapotranspiration depends linearly on the amount of water contained in a watershed, i.e.

$$
E T(t)=\gamma_{1} S(t)
$$

If for example, the amount of water in a region or the soil moisture increases the evapotranspiration will rise groundwater or the moisture of the soil increase than the evapotranspiration will rise. Similarly, the runoff function exhibits the following relationship

$$
r(t)=\gamma_{2} S(t)
$$

Inserting these two functions into the dynamic mass balance Eq. (2.1) yields

$$
\frac{d S(t)}{d t}=R(t)+P(t)-\gamma_{1} S(t)-\gamma_{2} S(t)-(1-h) x
$$

Let us assume that recharge and precipitation are constant over time, i.e., $R(t)=R_{0}$ and $P(t)=P_{0}$.

The introduced equations form a dynamic hydro-economic model. The intrinsic dynamic forces can be analyzed with the help of a so-called phase diagram, a graphical method to study the properties of dynamic systems. Figure 2.5 depicts the dynamic interrelations. To begin with, the periodic abstraction of a human settlement in the catchment area is represented by a horizontal line denoted by $(1-h) x$, where $x$ is the raw abstraction and $h \cdot x$ are the return flows after usage. In this simple model, we assume that water use of humans does not depend on the size of the local water stock $S(t)$. Hence, $(1-h) x$ is graphically represented by a horizontal line. The negatively sloped line in Fig. 2.5, shows the rate of replenishment of the water stock through inflows from precipitation, surface water and groundwater minus the outflows of surface and groundwater, as well as outflows through evapotranspiration (green water).

If the amount of replenished water is larger than the quantity of water used, i.e., $R_{0}+P_{0}-\left(\gamma_{1}+\gamma_{2}\right) S(t)>(1-h) x$ as indicated in Eq. (2.4), we can observe that the water stock will increase. Whether this is the case depends on the size of the water stock displayed on the horizontal axis. Let us assume that the current water stock is $S(t)=\Omega$, then the water stock will accumulate since $d S(t) / d t=$ $R(t)+P(t)-\left(\gamma_{1}-\gamma_{2}\right) S(t)-(1-h) x>0$. If $S(t)$ is somewhere on the right side of $S^{*}$, the reverse process takes place. This intrinsic dynamic behavior is identified by the arrows pointing to the intersection of both lines at $S^{*}$.

From Fig. 2.5, one cannot infer how long it will take until $S(t)$ reaches $S^{*}$, but it can be concluded that the stock will approach $S^{*}$. At the point where $S(t)=S^{*}$ holds, a hydro-economic equilibrium is reached, which is stationary in the sense that no further change of $S(t)$ will be observed. Additionally, $S^{*}$ is also stable, i.e., if 


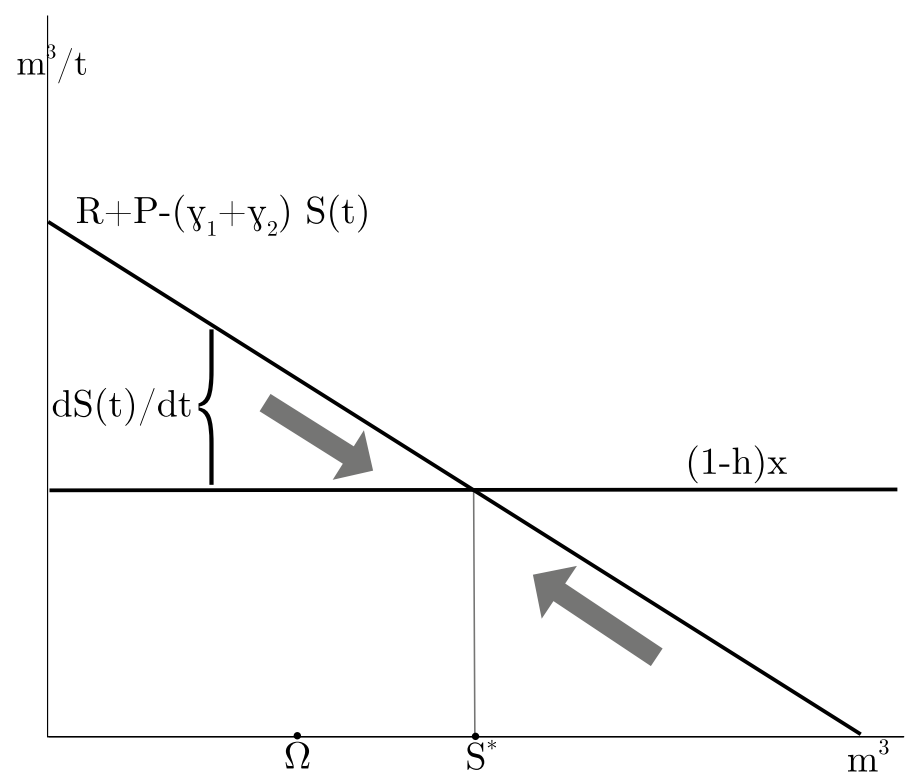

Fig. 2.5 Simple hydro-economic model. Source own illustration

$S(t)$ would deviate from $S^{*}$, let us say through a singular event like an unusual rain shower, then $S(t)$ would return to $S^{*}$ after a while. We call this state a steady-state equilibrium.

The question remains whether the water use $(1-h) x$ can be covered by the local water cycle, i.e., whether total water abstraction by the human settlement is sustainable. This depends on the level of net water abstraction $(1-h) x$. From Fig. 2.5, we can infer the equilibrium water stock level $S^{*}$ that corresponds to the quantity of water used by humans, i.e., $(1-h) x$. It follows that a higher level of water abstraction is associated with a smaller water stock in the local water cycle's equilibrium. Whether the water use is sustainable depends upon the critical threshold $\Omega$. This threshold depends on the whole ecological system and its interaction with the water cycle. We simply take this value as given. If the water stock $S(t)$ is less than $\Omega$, severe ecological damages will occur due to a decrease of basic stabilizing functions of water beyond its economic use: micro-climate stabilization, soil control, nutrient retention, supporting habitats and diversity, and flood control through wetlands. The corresponding upper bound of sustainable water abstraction can be calculated from Eq. (2.4) by setting $d S(t) / d t=0$ and solving for $x$. Inserting $S(t)=\Omega$ yields

$$
x^{\max }=\frac{R_{0}+P_{0}-g_{1} \Omega-g_{2} \Omega}{1-h}
$$

$x^{\max }$ is the upper bound of admissible water abstraction, implying that there is a quantity range $\left[0, x^{\max }\right]$ of sustainable water usage. If the human water utilization is less than the level $x^{\max }$, sustainability of the local water cycle is still assured. Of course, the change in the water table may lead to a change in the environment. But 
this change is not detrimental to the environment itself or its provision of ecological services nor to the people living in this catchment area. Box 2.2 describes a historic case of over-utilization of the water cycle with the help of the simple linear ecohydrological model.

\section{Box 2.2 The demise of the Mayas}

The Mayas dominated Middle America for at least 1500 years and suddenly, around the ninth century A.D., their civilization vanished within a very short time. It is estimated that in the pre-Columbian time over 19 million people lived in Meso-America, and that after the ninth century only 10 percent were left. Archeologists and historians puzzled about the reasons for this sudden demise of this ancient civilization. Numerous explanations were presented, such as epidemic disease, warfare, and overpopulation. Today, there is reason to believe that severe droughts have caused the collapse of the agricultural system, and hence destroyed the livelihood of the Mayas. These droughts were not only the result of a long wave periodic change of the climate, but they also resulted from the deforestation that took place to gain more farmland. Dr. Thomas L. Sever, an archeologist with NASA's Marshall Space Flight Center, said that the rise of droughts in this area could be traced back to the Mayas themselves. In some recent studies, geophysicists developed complex hydrological and climatological models to reconstruct the impact of deforestation on the local climate.

Sources: Cook et al. (2012), Kuil et al. (2016)

\section{$2.4 \quad$ Exercises}

\section{Exercise 2.1 Water availability in the 2020s}

The source we use to describe the water availability on earth is the best one available, but it is over three decades old. Try to find reliable sources to update the values for the major categories, such as total water reserves, total seawater, freshwater, glaciers, etc., to the current times, i.e., the 2020s. Are there differences to be observed? If yes, what could be reasons for this? Is the literature unified on this issue, or are there controversies?

\section{Exercise 2.2 The demise of Mayas}

We can use our simple linear model to get an idea of how various factors were at work and led to the decline of the agricultural base as a result of increased deforestation. Our approach focusses on some pivotal interactions that cause the detrimental effects 
of deforestation. To do so we extend Eq.(2.1), by introducing a coefficient $\beta$ that indicates the capacity of the local climate system to return evapotranspiration as precipitation.

$$
\frac{d S(t)}{d t}=R(t)+P(t)-(1-\beta) E T-r-(1-h) x
$$

were $0 \leq \beta<1$. This coefficient captures various climatological effects that are responsible for the creation of clouds through local evapotranspiration identified by climatologists: The surface albedo effect, aerodynamic effects, and chemical effects, to name some. Let us confine to the surface albedo effect. Albedo is the ratio between reflected radiation to incident solar radiation. The higher the albedo the less radiation (energy) is absorbed from the earth. The albedo rises with the deforestation because cultivated land reflects more radiation. With rising albedo, the absorption of energy from radiation decreases, which leads to less heat flux. Less heat energy causes less vapor production and results in a decrease of cloud building. Less clouds are associated with less precipitation.

This transmission chain is captured by $\beta$, which depends on deforestation. Let $F$ be total land available in an area. This land is either covered by forest or it is utilized as cropland, whereas the latter case is denoted by $A$. Thus $\beta=\beta(A)$ with $\beta^{\prime}(A)<0$.

In addition, we have to distinguish between forest evapotranspiration and cropland evapotranspiration. Extending the linear model leads to

$$
\begin{aligned}
& E T_{1}(t)=\gamma_{1} S(t)(F-A) \\
& E T_{2}(t)=\gamma_{2} S(t) A
\end{aligned}
$$

where Eqs. (2.7) and (2.8) represent the evapotranspiration of forest and of cropland, respectively. We assume, that $\gamma_{1} \geq \gamma_{2}$. Finally, runoff is given by

$$
r(t)=\gamma_{3} S(t) A
$$

assuming that runoff takes place mainly in the cultivated areas. We also assume, that $\gamma_{1}<\gamma_{2}+\gamma_{3}$, i.e., an increase of cropland A leads to an increase of evapotranspiration. If we insert these three equations into Eq. (2.6) we get

$$
\frac{d S(t)}{d t}=R(t)+P(t)-(1-\beta)\left(\gamma_{1} S(t)(F-A)+\gamma_{2} S(t) A\right)-\gamma_{3} S(t) A-(1-h) x
$$

Agricultural production depends on water availability $S(t)$ and, of course, on the area $A$. Let us assume the simple production function

$$
C=\frac{A}{F} \operatorname{Max}[\delta(S(t)-\Omega), 0]
$$

where $\delta$ is agricultural productivity. Output depends not only on the area cultivated but also on the amount of water available. This function depicts the inherent hydrological 


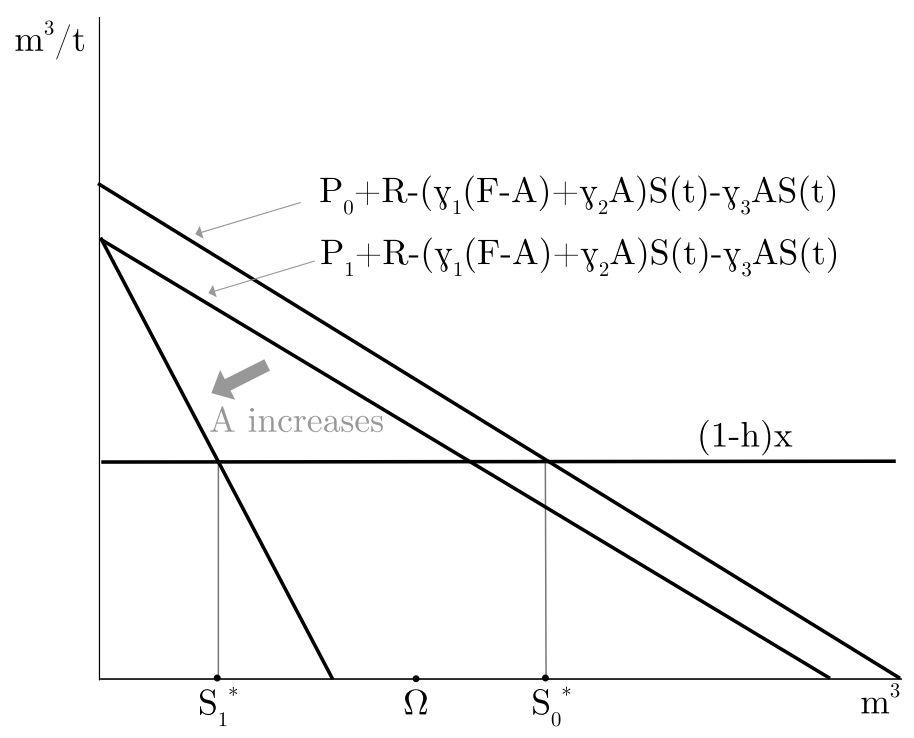

Fig. 2.6 The demise of the Maya. Source own illustration

and ecological preconditions of agricultural production in an extreme manner. If the water stock is above a critical threshold, agricultural production is possible. If $S$ falls short of $\Omega$ the whole production breaks down. Figure 2.6 shows the problem of increased deforestation.

In the course of an exogenous decrease of precipitation from $P_{0}$ to $P_{1}$ the $d S / d t$ curve shifts downwards and the output of agriculture drops (see Eq. (2.11)). The Maya react with expanding cropland because they try to compensate the decreased productivity of cropland by increasing the size of it (see Eq. (2.11)). As a result, the increased deforestation leads to a clockwise rotation of the graph reflecting Eq. (2.10). The final hydrological equilibrium is $S_{1}^{*}$ which is located to the left of $\Omega$ leading to severe crop failures, and finally to the demise of the Mayas.

\subsection{Further Reading}

A good overview about the water availability and water cycle is given by Shiklomanov (1990). More details about the components of the water balance could be found in special books which focus on meteorology or hydrology such as Brutsaert et al. (2005), Gordon et al. (2004), as well as Holton and Hakim (2012). Introductory references to geohydrological topics and groundwater are Karamouz et al. (2011) and Thangarajan (2007). 


\section{References}

Brutsaert, W., \& others. (2005). Hydrology: An introduction. Cambridge [Cambridgeshire]; New York: Cambridge University Press.

Cook, B. I., Anchukaitis, K. J., Kaplan, J. O., Puma, M. J., \& Kelley, M. (2012). Pre-Columbian deforestation as an amplifier of drought in Mesoamerica. Geophysical Research Letters, 39(16), $1-5$.

Gordon, N. D., McMahon, T. A., Finlayson, B. L., Gippel, C. J., \& Nathan, R. J. (2004). Stream hydrology: An introduction for ecologists. New Jersey: Wiley.

GWP. (2000). Integrated water resources management. Technical advisory committee (TAC) background paper no. 4. Stockholm, Sweden: Global Water Partnership.

Holton, J. R., \& Hakim, G. J. (2012). An introduction to dynamic meteorology (Vol. 88). Cambridge: Academic.

Houghton, J. (2004). Global warming: The complete briefing (3rd ed.). Cambridge: Cambridge University Press.

Karamouz, M., Ahmadi, A., \& Akhbari, M. (2011). Groundwater hydrology: Engineering, planning, and management. London, UK: CRC Press.

Kuil, L., Carr, G., Viglione, A., Prskawetz, A., \& Blöschl, G. (2016). Conceptualizing sociohydrological drought processes: The case of the Maya collapse. Water Resources Research, 52(8), 6222-6242.

Shiklomanov, I. A. (1990). World fresh water resources. Pages 13-24 of: Water in crisis: A guide to the world's fresh water resources. In: Gleick, P. H. (Ed.), Water in crisis: A guide to the world's fresh water resources. New York: Oxford University Press.

Thangarajan, M. (2007). Groundwater: Resource evaluation, augmentation, contamination, restoration, modeling and management. New York City, NY, U.S.A.: Springer Science \& Business Media.

Open Access This chapter is licensed under the terms of the Creative Commons Attribution 4.0 International License (http://creativecommons.org/licenses/by/4.0/), which permits use, sharing, adaptation, distribution and reproduction in any medium or format, as long as you give appropriate credit to the original author(s) and the source, provide a link to the Creative Commons license and indicate if changes were made.

The images or other third party material in this chapter are included in the chapter's Creative Commons license, unless indicated otherwise in a credit line to the material. If material is not included in the chapter's Creative Commons license and your intended use is not permitted by statutory regulation or exceeds the permitted use, you will need to obtain permission directly from the copyright holder.

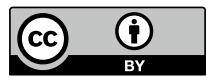

